\title{
EchoGéo
}

$33 \mid 2015$

Activisme, participation, contestation : la place des habitants dans les processus de patrimonialisation en périphéries urbaines

\section{Politiques de valorisation patrimoniale et figuration des habitants en banlieue parisienne (Plaine Commune)}

\section{Sébastien Jacquot}

\section{(2) OpenEdition}

\section{Journals}

Electronic version

URL: https://journals.openedition.org/echogeo/14317

DOI: 10.4000/echogeo.14317

ISSN: 1963-1197

\section{Publisher}

Pôle de recherche pour l'organisation et la diffusion de l'information géographique (CNRS UMR 8586)

\section{Electronic reference}

Sébastien Jacquot, "Politiques de valorisation patrimoniale et figuration des habitants en banlieue parisienne (Plaine Commune)", EchoGéo [Online], 33 | 2015, Online since 30 September 2015, connection on 11 August 2021. URL: http://journals.openedition.org/echogeo/14317 ; DOI: https:// doi.org/10.4000/echogeo.14317

\section{This text was automatically generated on 11 August 2021}

EchoGéo est mis à disposition selon les termes de la licence Creative Commons Attribution - Pas d'Utilisation Commerciale - Pas de Modification 4.0 International (CC BY-NC-ND) 


\title{
Politiques de valorisation patrimoniale et figuration des habitants en banlieue parisienne (Plaine Commune)
}

\author{
Sébastien Jacquot
}

\section{Introduction}

1 Plaine Commune, communauté d'agglomération au nord du périphérique parisien, en Seine-Saint-Denis, a obtenu le label Ville d'art et d'histoire fin 2013. La nouvelle animatrice du patrimoine, recrutée fin 2014, était auparavant chargée de la valorisation de la cité-jardin de Stains, haut-lieu de la politique patrimoniale de Plaine Commune. Cette politique patrimoniale est fondée à la fois sur un patrimoine distinct du patrimoine monumental parisien, emblématique d'une banlieue populaire et anciennement industrielle, et sur une façon alternative de valoriser le patrimoine, en relation avec le tissu local, tranchant avec les valorisations précédentes centrées uniquement sur son patrimoine le plus monumental, telle la Basilique de Saint-Denis.

Quelle(s) place(s) ces politiques de valorisation du patrimoine confèrent-elles aux habitants? Qui sont les destinataires de ces politiques de valorisation? Présentées comme touristiques, tournent-elles le dos aux habitants? Or à Plaine Commune les politiques touristiques révèlent des enjeux qui débordent la question de l'attraction de visiteurs extérieurs, positionnant l'habitant comme bénéficiaire et visiteur. De plus, du point de vue du contenu de ces politiques, le passage du monumental au patrimoine de banlieue institue les habitants en témoins et signifiés du récit patrimonial. Enfin, du point de vue de la production des politiques patrimoniales, les discours publics de la participation et mobilisation habitante configurent aussi les habitants comme sujets. Les habitants sont donc invités à endosser différents rôles, délimités par des modes de rapport aux institutions et des capacités d'agir. Ces rôles semblent aussi brouiller des 
séparations établies, entre touristes et habitants, entre habitants et institutions. Il ne s'agit pas d'essentialiser la distinction entre touristes et habitants - plusieurs travaux ont montré l'intérêt d'une remise en cause de cette catégorisation binaire (Sherlock, 2001 ; Stock, 2005), mais plutôt de voir comment dans les discours locaux ces catégories sont mobilisées et redéfinies.

3 L'objectif de cet article est ainsi de caractériser la façon dont les institutions en charge de la valorisation patrimoniale définissent les rôles tenus par les habitants en lien avec le patrimoine. Cette construction de l'habitant comme figure des politiques patrimoniales sera étudiée par le détour des politiques touristiques en banlieue, à Plaine Commune.

4 Le patrimoine est appréhendé ici comme le résultat d'un processus social, culturel, économique : la patrimonialisation (Veschambre, 2007). Après une discussion du rôle configurant des politiques patrimoniales, le second temps est consacré à la politique de valorisation touristique du patrimoine monumental mené à Saint-Denis entre 1960 et 1980, par des habitants agissant à travers un syndicat d'initiatives. Cela permettra de faire ressortir la rupture des années 1990 et 2000, marquée par la naissance d'une action publique intercommunale plus volontariste valorisant de nouveaux patrimoines. La troisième partie montre la façon dont cette rupture produit des figurations multiples des habitants. Enfin sont examinées les conséquences sur l'action publique de la valorisation patrimoniale de ces figurations.

5 Cette recherche s'appuie sur une exploration des archives municipales de Saint-Denis ${ }^{1}$, sur des entretiens menés avec des acteurs publics et privés de la patrimonialisation et mise en tourisme du territoire, sur une étude des documents publics énonçant ces politiques et sur de l'observation participante de diverses réunions et manifestations publiques ou semi-publiques.

\section{Le rôle configurant des politiques patrimoniales}

\section{Patrimoine et habitants}

Alors que des témoignages anciens évoquent l'indifférence des habitants à l'égard du patrimoine (Poulot, 2006), les habitants deviennent les sujets des mobilisations et politiques patrimoniales. La production patrimoniale n'est plus seulement un monopole d'État, de «nouveaux acteurs » font leur entrée dans le champ patrimonial à la faveur des "mobilisations patrimoniales", à partir de la fin des année 1970. Ils interviennent en tant que "non-institutionnels, habitants, citoyens » (Veschambre, 2008), souvent par le prisme associatif (Glevarec, Saez, 2002).

7 Ces mobilisations habitantes peuvent être portées par des passions jugées parfois débordantes (Le Goff, 1998), qui interpellent les institutions en charge de la patrimonialisation. Le patrimoine y est souvent articulé à un registre intime et familier, y compris dans le cas d'un patrimoine monumental consacré comme, par exemple, le château de Lunéville (Pecqueux, Tornatore, 2013). Ces émotions deviennent politiques en ce qu'elles donnent forme à des schémas d'action (Fabvre, 2013), se cristallisant sur des conflits qui portent sur le pouvoir de "décréter le patrimoine » (Veschambre, 2008), comme dans les luttes autour des patrimoines dissonants (Ashworth, Tunbridge, Graham, 1999; Chivallon, 2002). Dans ces mobilisations patrimoniales, les habitants sont posés comme extérieurs aux institutions, en opposition ou en résistance à des 
modes dominants de patrimonialisation ou à des destructions et indifférences patrimoniales.

Dans le cadre des dynamiques collaboratives et participatives en revanche, la place des habitants est davantage institutionnalisée: H. Glevarec et G. Saez (2002) précisaient déjà qu'on ne pouvait opposer frontalement associations et institutions. La diffusion d'une «injonction participative» (Sintomer, Blondiaux 2002) ou d'un "paradigme participatif » (Bacqué, Rey, Sintomer, 2005) se traduisent par la mise en place d'outils d'implication des citoyens dans les politiques urbaines, et en particulier patrimoniales. Cela suppose la reconnaissance d'une capacité des citoyens à identifier des éléments patrimoniaux et des modalités de valorisation, compétences plus seulement dévolues aux experts du patrimoine, et justifiant le développement de mécanismes participatifs, souvent qualifiés de concertation. Sous diverses formes, ces participations institutionnalisées se retrouvent dans la gestion des secteurs sauvegardés en France, des centres historiques de certaines villes d'Amérique Centrale (Orellana, 2006), ou encore dans le cadre de la politique patrimoniale de la ville de Paris entre 2001 et 2007 (Poupeau, 2009). Même l'UNESCO désormais encourage depuis 2007 le développement de mécanismes participatifs pour la gestion des biens du Patrimoine mondial (GravariBarbas, Jacquot, 2014).

9 Toutefois, dans cette présentation des politiques de valorisation patrimoniale à Plaine Commune, il ne s'agit de montrer ni des mobilisations et résistances habitantes, ni des participations et intégrations effectives aux politiques patrimoniales. Le questionnement est décentré sur les discours tenus sur les habitants, dans le cadre des politiques patrimoniales. De quelle façon les habitants constituent-ils les sujets démultipliés et en même temps non nécessairement présents de la valorisation du patrimoine en banlieues?

\section{Figuration des sujets de l'action et de la réception patrimoniale}

10 Ce travail s'inscrit dans une approche des politiques publiques qui en considère les dimensions symboliques. Suivant la relecture opérée par D. Cefai (2007) des travaux sur les comportements collectifs et mouvements sociaux, les dynamiques publiques et collectives font apparaitre « des lieux et des moments, des personnes et des artefacts », bref « configurent $d u$ sens ». Cette perspective fait écho aux travaux de J. Rancière, articulant politique, constitution des collectifs et «constitution symbolique du social» (2004). Cette solidarité entre politique et identification des configurations socio-spatiales est traduite par la notion de "partage du sensible», qui désigne "ce système d'évidences sensibles qui donne à voir en même temps l'existence d'un commun et les découpages qui y définissent les places et les parts respectives " (Rancière, 2000). Dès lors peut être étudiée une certaine "esthétique du politique» (Rancière, 2000), qui fixe les places, les compétences et incompétences. S'inspirant de J. Rancière, certains travaux ont porté leur attention sur les légitimités ou illégitimités à participer et prendre part au politique, à travers les processus participatifs (Fourniau, 2007) ou dans le cadre de la politique de la ville en France (Diçek, 2007).

11 Or les politiques de valorisation patrimoniale impliquent aussi un choix sur ce qui fait collectif, comme le montrent les controverses patrimoniales, révélant des enjeux politiques. Dans Le spectateur émancipé, J. Rancière (2008) montre que les "formes sensibles de l'expérience» et la constitution d'une communauté se jouent dans les 
différents discours sur la place et le rôle des publics du spectacle théâtral (de Platon à Brechet), à partir des oppositions actif - passif, regard - action.

Parallèlement, les politiques patrimoniales configurent des formes de l'expérience (Jacquot, 2009), en mettant l'accent sur la façon dont y est défini l'habitant, sa place et ses capacités d'agir. Portées aussi par des enjeux de reconnaissance, les dynamiques de patrimonialisation positionnent la notion d'habitant comme " une figure produite au gré des luttes de classement indigènes " (Chavanon, 2014). Il ne s'agit pas uniquement de qualifier les habitants en tant que référencés par les politiques patrimoniales et intégrés dans une histoire et un territoire particuliers. Ces politiques patrimoniales ne posent pas seulement les habitants comme leur extérieur auxquelles elles donnent une signification, mais impliquent aussi les habitants de différentes façons : bénéficiaires, sujets dont il est question, mais aussi participants. Autrement dit, ces politiques ne désignent pas seulement les habitants, elles dessinent aussi leur registre attendu d'action et de modes de relation avec les institutions.

La " figure », résultat d'un processus de figuration, renvoie aux façons dont sont définis, délimités et qualifiés les habitants. Plusieurs travaux sur la participation mobilisent cette notion. Ainsi lors des controverses liées à l'implantation d'éoliennes en France (Le Floch, 2011), les acteurs catégorisent des publics et "esquissent des figures " des participants, entre citadin, riverain, habitant, comme autant de façon de penser le rapport au projet, et partant la légitimité à participer aux discussions (par exemple le riverain est "une figure construite de manière à être mise à l'écart »). Via les dispositifs de concertation, «l'individu-destinataire est construit, c'est-à-dire figuré et projeté dans les actes et par les discours qui le prennent en compte ", révélant plusieurs figures de l'habitant (Noyer, Raoul, 2008).

De même, l'étude des processus participatifs et discours politiques révèle au sein du champ politique un discours de la demande sociale, mobilisant notamment une construction de l'« habitant» et de ses attentes, permettant de présenter les politiques publiques comme une réponse à une telle demande (Anquetin, Freyermuth, 2009), et de faire apparaître les catégorisations et différenciations opérées entre habitants (Biland, 2006). Dès lors cette configuration de l'«habitant» et de ses modalités d'agir politiquement relève d'une " offre de participation » (Petit, 2014). En interrogeant deux périodes - les années 1960 à 1980, et les années 2000 et 2010 - quelles figures et définitions d'une modalité de l'agir comme habitant produisent les démarches de valorisation patrimoniale par le tourisme à Plaine Commune?

\section{Valorisation patrimoniale par le tourisme : du syndicat d'initiatives à la politique communautaire}

La naissance des offices de tourisme et syndicats d'initiatives correspond à une première forme de rapports entre politiques de valorisation du patrimoine et habitants en banlieue parisienne. L'exploitation des archives communales de la commune de Saint-Denis permet d'étudier la généalogie des politiques de valorisation patrimoniale par le tourisme et le rôle joué par une partie des habitants dans une commune connaissant des pratiques touristiques depuis le $\mathrm{XIX}^{\mathrm{e}}$ siècle, exclusivement en raison de la Basilique de Saint-Denis, nécropole royale (Leniaud, 1996). 


\section{Naissance d'une politique touristique et habitants à Saint-Denis}

L'office de tourisme de Saint-Denis existe depuis 1936. Il a été créé dans l'urgence en vue de l'exposition universelle de 1937, avec le statut de syndicat d'initiatives, espérant " $y$ attirer des étrangers", en mettant en avant ses ressources patrimoniales les plus classiques : la Basilique de Saint-Denis et le musée de Saint-Denis (actuel Musée d'art et d'histoire), fondé en 1901. Avant cette création, les acteurs locaux du patrimoine effectuaient de fait des missions touristiques : par exemple le conservateur du musée était l'interlocuteur des maisons d'édition de guides (type Baedeker).

Ce syndicat d'initiatives, disparu durant la seconde guerre mondiale, réapparaît en 1966, avec un statut associatif. La municipalité, qui a encouragé son développement, le finance en grande partie via une subvention annuelle. Les habitants qui composent le syndicat d'initiatives, dont chaque compte-rendu de réunion fournit la liste, proviennent plutôt des couches moyennes et supérieures de la ville : notaire, médecin, enseignant, chanoine de la Basilique Saint-Denis, employée du Conseil Général, etc.

De nombreuses actions sont marginales par rapport à la question de la valorisation patrimoniale : comité de fleurissement, organisation du bal annuel et promotion des autres destinations touristiques françaises (au départ la fonction dominante du syndicat d'initiatives de Saint-Denis est davantage d'organiser les vacances de ses habitants que d'attirer des touristes). Toutefois, plusieurs initiatives touristiques de valorisation du patrimoine sont développées : sous couvert d'une politique touristique associative, dans les années 1960 et 1970, se trouvent engagés des débats et actions permettant de définir des priorités en terme de développement et valorisation du patrimoine, en lien avec les acteurs publics, notamment la municipalité.

\section{Un engagement patrimonial classique}

Des années 1930 aux années 1980, une forte continuité caractérise le patrimoine mis en avant et valorisé par le syndicat d'initiatives, centré sur ses manifestations les plus classiques: la Basilique et le musée, et progressivement la maison de la Légion d'honneur. L'engagement des habitants composant le syndicat d'initiatives se porte sur la mise en valeur de ce patrimoine classique, sans remettre en cause sa préséance : pas de trace dans les comptes rendus de mobilisations ou d'indignations en faveur d'un patrimoine alternatif. La Basilique focalise l'énergie des membres du syndicat d'initiatives.

20 Ainsi un projet de son et lumière de la Basilique est proposé en 1972 par le syndicat d'initiatives. Ces actions habitantes s'inscrivent en continuité avec les objectifs de valorisation patrimoniale menée par la municipalité. La Basilique étant gérée par la Caisse des Monuments Historiques, une convention est envisagée entre les deux organismes, mais les discussions traînent. Le projet de son et lumière est abandonné. Un accord est néanmoins établi pour l'organisation de visites guidées au sein de la Basilique dans le cadre d'une convention Villes d'art et Cités d'art et d'histoire, lancée en 1967 par la Caisse Nationale des Monuments Historiques et des Sites. Il s'agit de l'ancêtre du label Ville et Pays d'Art et d'Histoire (obtenu par Plaine Commune en 2013) ${ }^{2}$. Les travaux de rénovation urbaine autour de la Basilique sont également l'occasion d'un engagement accru du syndicat d'initiatives vers l'animation urbaine, en 
proposant un aménagement de la station de métro Basilique de Saint-Denis, ouverte en 1976, sur le modèle de celle du Louvre.

21 L'idée d'un patrimoine de banlieue n'est alors pas présente. L'Union départementale et régionale des Syndicats d'initiatives et offices de tourisme d'Ile-de-France favorise une mise en réseau des initiatives touristiques en périphérie de Paris. Le discours est toutefois très différent de celui d'aujourd'hui : les enjeux perçus comme communs sont ceux de la redécouverte de richesses méconnues, de trésors de l'Histoire nationale, rendus moins visibles par leur localisation ${ }^{3}$. Ce n'est pas l'idée d'une continuité fondée par une même condition de banlieue parisienne qui prévaut. Cette action de valorisation par le syndicat d'initiatives, portée par les franges notables de la population de Saint-Denis, demeure donc très classique dans ses appréhensions patrimoniales les plus visibles.

\section{De timides diversifications}

Quelques timides diversifications apparaissent néanmoins. Sur chaque dépliant, brochure, discours, émanant du syndicat d'initiatives, il est rappelé que Saint Denis est aussi une ville active, une cité industrielle (sans que des politiques de valorisation prennent en charge cet aspect). Une brochure préparée en 1976 mentionne simplement "l'industrie d'une ville au travail ». Les dimensions populaires, ouvrières, industrielles, ne sont pas affichées comme thèmes de valorisation. Les seules ouvertures thématiques sont sollicités par la commune, lorsqu'elle reçoit des délégations soviétiques, d'Ouzbékistan par exemple, ou des membres du PC italien, auxquelles elle fait visiter les réalisations du communisme municipal (écoles, crèche, équipements culturels). Elle charge alors le syndicat d'initiatives d'en assurer les visites, ce qui ne semble pas ravir ses membres.

En fait, la diversification des types de patrimoine valorisés émane davantage de la commune que des habitants, et va de pair avec la naissance d'un discours porté sur le rôle des habitants. Dès 1979, un rapport au bureau municipal sur les modalités de la rénovation urbaine mentionne à la fois une diversification des potentialités patrimoniales et le rôle des habitants :

«À l'inverse de la grande majorité des communes de banlieue, Saint-Denis garde son unité urbaine et les Dionysiens se sentent habitants et non transitaires comme dans les cités dortoirs. Le fait que Saint-Denis a un passé historique et une densité industrielle importants explique cette réalité. Réalité qui est accrue par les traces de l'histoire que conserve Saint Denis sur son territoire soit sous la forme de monuments (Basilique, Carmel, Couvent des Ursulines, Maison des Arbalétriers, caves, séchoirs, moulins et usines anciens...), soit sous la forme de voirie (tracé des rues médiévales), des cours d'eau (...) »4.

Plus tard, en 1995, le Plan de signalétique et d'interprétation de la ville de Saint- Denis, préparé par un cabinet conseil, énonce que «l'Histoire est le principal atout touristique de la ville. Ceci est illustré par la Basilique, les fouilles archéologiques, le Musée d'Art et d'Histoire, les archives municipales, sans oublier le potentiel de témoignages des populations locales». Deux figures d'habitant émergent de ces deux documents : l'habitant comme témoin ; l'habitant comme porteur d'un rapport particulier d'appropriation au territoire. Dans ces deux cas, l'habitant demeure en position d'extériorité par rapport aux politiques menées, dont il est bénéficiaire et contributeur externe. 
25 Au final, cette période est marquée par une valorisation patrimoniale centrée sur un patrimoine national et prise en charge par le syndicat d'initiatives, via des habitants volontaires, en interaction avec la municipalité. Cette forme classique de valorisation renvoie peu les échos d'une ville industrielle et de ses enjeux. La dimension touristique y apparaît commune à de nombreux autres territoires : son objectif est bien d'attirer des visiteurs, qui deviendront "nos meilleurs ambassadeurs aux quatre coins du monde", selon la directrice de l'OT lors d'un discours en $1979^{5}$. Cette notion d'ambassadeur va connaître un spectaculaire renversement à partir des années 2000. Il témoigne des mutations des objectifs de la valorisation patrimoniale en même temps que des nouvelles figures attribuées aux habitants, en lien avec les politiques patrimoniales et touristiques élaborées dans le contexte de construction intercommunale d'un projet local de développement.

\section{Le tournant de la valorisation patrimoniale : Plaine Commune et le tourisme}

26 Le tournant de la valorisation patrimoniale s'inscrit au croisement de plusieurs dynamiques: reconversion territoriale et fonctionnelle post-désindustrialisation, changement d'échelle - de Saint-Denis à Plaine Commune, intérêt pour de nouvelles formes patrimoniales, articulation du patrimoine et du tourisme à un projet de développement local, et plus récemment débats sur les formes de l'intégration métropolitaine (Braouezec, 2012).

Ce territoire anciennement industriel de la banlieue rouge parisienne (Brunet, 1980) a connu un processus de désindustrialisation important, avec par exemple la fermeture de 103 grandes entreprises entre 1968 et 1980 à La Plaine (commune de Saint-Denis) ${ }^{6}$. Les élus ont réagi à ces mutations territoriales avec la création en 1985 d'un syndicat intercommunal, Plaine Renaissance, creuset de la communauté d'agglomération Plaine Commune, formée en 2001, qui regroupe 9 communes depuis l'adhésion de Saint-Ouen en 20137. La revitalisation économique est au cœur du projet territorial (Bertho, 2008), marquant des stratégies pragmatiques des élus locaux face aux mutations territoriales (Bellanger, Mischi, 2013). Elle est marquée par la reconversion des friches industrielles au profit de l'immobilier d'affaires (Malézieux, 2003), ou des investissements des grandes chaînes hôtelières (Decelle, Jacquot, 2014), encouragés par les acteurs publics locaux et métropolitains, profitant de la congestion parisienne.

Déconnecté de ce développement hôtelier de chaîne, pour des touristes qui visitent peu le territoire de la Seine-Saint-Denis, mais profitent des bonnes connections métropolitaines, notamment depuis les infrastructures en lien avec la Coupe du Monde 1998, Plaine Commune mène une politique de valorisation patrimoniale, par le détour du tourisme. En effet, la valorisation patrimoniale s'inscrit de façon croissante dans des politiques de mise en tourisme (Tomas, 2004), en lien avec l'argument du développement local (Cousin, 2007 ; Violier, 2008), et ce territoire de la banlieue parisienne n'échappe pas à ce mouvement.

Dès 1993, le député-maire Patrick Braouezec publie le Livre Blanc du tourisme, énonçant l'apport du tourisme au développement local. L'horizon de la Coupe du Monde accélère la structuration d'une politique intercommunale de valorisation touristique du patrimoine. La compétence tourisme devient communautaire en 2002, parallèlement à l'office de tourisme, et une chargée de mission (Th. Kouloumbri) est recrutée pour 
réaliser un Schéma touristique communautaire, pour la période 2003-2008. Ce document stratégique de valorisation du patrimoine en élargit la signification et les enjeux: traces industrielles, voies fluviales, jardins ouvriers, apparaissent désormais comme "richesses patrimoniales». Ainsi, cette valorisation ne concerne plus seulement le patrimoine monumental et historique telle la Basilique. Un des projets emblématiques de ce premier Schéma concerne la mise en tourisme de la cité-jardin de Stains, édifiée en 1921 et 1933 pour le compte de l'office public d'HBM de la Seine, suivant les principes développés par Ebenezer Howard, et encore parc de logement social actuellement. L'accent est davantage mis sur ce qui constitue spécifiquement un patrimoine de banlieue (Jacquot, Gravari-Barbas, Fagnoni, 2014), c'est-à-dire des éléments patrimoniaux qui donnent de la consistance à la catégorie de banlieue et à son histoire, à la différence de la Basilique, patrimoine localisé en banlieue.

Cette démarche de valorisation du patrimoine en banlieue n'est pas spécifique à Plaine Commune, mais concerne d'autres périphéries métropolitaines, des quartiers nord de Marseille ${ }^{8}$ à l'East End à Londres ou les cités du modernisme, inscrites sur la Liste du Patrimoine mondial, dans les périphéries de Berlin. Elle s'appuie sur l'émergence de nouvelles formes patrimoniales dont des témoignages importants se trouvent en périphérie, au croisement de démarches expertes d'inventaire et de mobilisations locales, en dépit des démolitions (Veschambre, 2008): patrimoine industriel, patrimonialisation des réalisations du logement social au $\mathrm{XX}^{e}$ siècle (Duchêne, Langumier, Morel Journel, 2013), des HBM (comme la cité 212 au Blanc-Mesnil) et citésjardins aux réalisations de la seconde moitié $d u X X^{e}$ siècle, portées par le nom d'un grand architecte tel Le Corbusier à Firminy (Guillot, 2008), ou en Seine-Saint-Denis autour des réalisations d'André Lurçat ${ }^{9}$. Cette patrimonialisation s'articule aux démarches mémorielles (Rautenberg, 2003), articulées aux politiques de renouvellement urbain, et entraîne notamment des « démarches de valorisation de la mémoire de l'immigration " (Chavanon, 2014), y compris par le biais du tourisme (Chapuis, Jacquot, 2015). Enfin, ces politiques ont également une portée symbolique, le patrimoine servant à la " production de récits identitaires » au service de la promotion d'une image de la localité (Nicolas, Zanetti, 2013). En banlieue parisienne le discours du nécessaire changement d'image du territoire est récurrent, opéré par la valorisation touristique du patrimoine (Lazzarotti, 2004), comme un prolongement sous une autre forme des politiques culturelles contemporaines de la construction de la banlieue rouge, «capable(s) de retourner la stigmatisation attachée aux banlieues ouvrières " selon A. Fourcaut (2007).

31 La politique de valorisation patrimoniale devient ainsi une façon de dire une spécificité territoriale, en rupture avec la centralité parisienne : cette catégorie de « patrimoine de banlieue » n'est pas seulement un outil d'analyse, elle est revendiquée par ces nouveaux acteurs à Plaine Commune (Kouloumbri, 2004). L'expérience de Stains, jugée réussie, débouche sur un projet de mise en réseau touristique des cités-jardins en petite couronne. Parallèlement, de nouvelles thématiques patrimoniales sont développées : patrimoine industriel, patrimoine immatériel, patrimoine maraîcher, comme autant de façons de lire l'histoire du territoire. L'obtention du label Ville d'art et d'histoire en 2013 consacre cette politique.

32 Ainsi deux logiques différentes et non articulées de développement touristique en banlieue coexistent sur le même territoire ${ }^{10}$. Elles ne relèvent pas des mêmes services, et surtout ne visent pas les mêmes "clientèles" ou "visiteurs ». Le développement 
hôtelier s'inscrit d'emblée dans une logique métropolitaine, dans des espaces bien connectés au centre métropolitain, tandis que les politiques touristiques sont une politique de valorisation patrimoniale articulée à une mise en récit du territoire (traces de l'industrie, du maraîchage, des histoires sociales), et s'adresse à des visiteurs composites, notamment locaux et Franciliens, loin d'un public exclusivement touristique.

Alors que cette politique de valorisation patrimoniale repose sur la volonté d'un développement local et une réévaluation des histoires locales, quelle place y est proposée aux habitants?

Illustration 1- Dynamiques de valorisation patrimoniale et touristique à Plaine Commune, 1960-2015

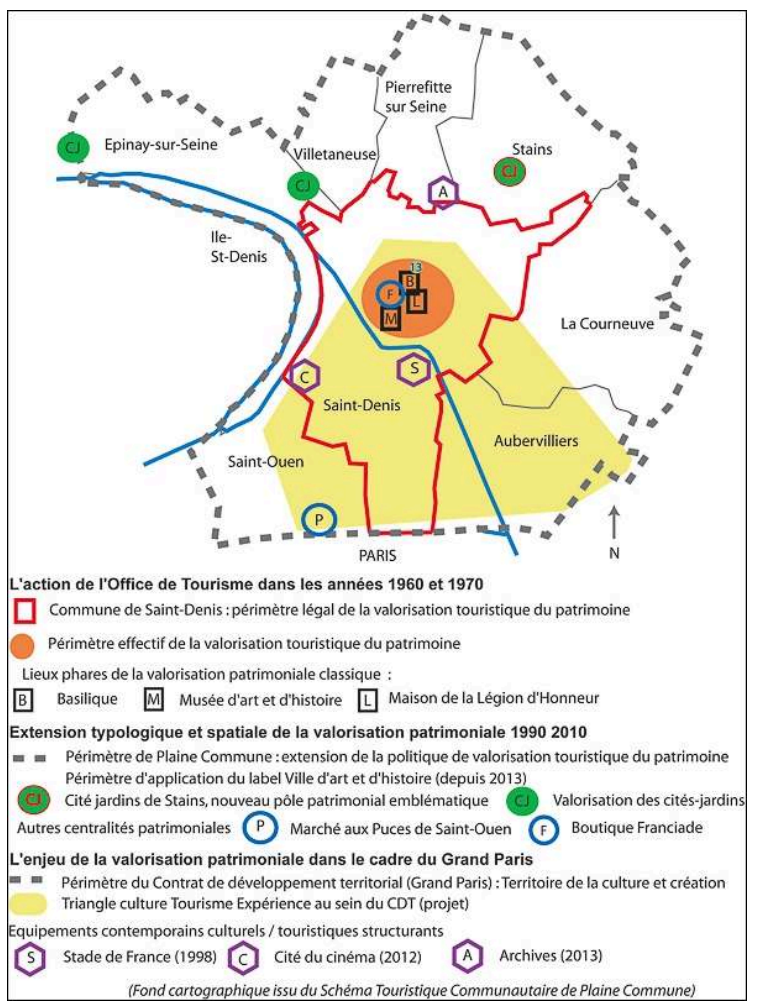

Auteur: S. Jacquot, 2015.

\section{Les figures habitantes à travers les politiques de valorisation patrimoniale à Plaine Commune}

Les politiques de valorisation patrimoniale menées par Plaine Commune dessinent différentes figures de l'habitant, en lui attribuant des rôles.

\section{Les destinataires d'une politique de valorisation touristique du patrimoine}

P. Braouezec, ancien député-maire de Saint-Denis et président de Plaine-Commune, revenait à l'occasion d'une réunion sur le tourisme urbain en $2012^{11}$ sur l'importance de la politique touristique intercommunale. Les questions de fierté et dignité ponctuaient 
son discours. La valorisation patrimoniale par le tourisme constitue selon lui un outil de marketing territorial, à la fois externe pour inverser les images médiatiques négatives associées à ce territoire, mais aussi interne pour produire un sentiment de fierté et d'appartenance et revaloriser les identités locales.

Dans ce même discours il mentionne les habitants de deux autres façons, toujours articulées à l'échelle locale. Tout d'abord ceux-ci sont questionnés dans leur réception du patrimoine le plus classique. À un possible étonnement face à des familles d'origine étrangères venues visiter les fouilles de la Basilique, qui ne s'expliquerait qu'en référence à une conception nationalo-centrée dépassée de ce patrimoine (celle de l'expression "nos ancêtres les Gaulois »), il oppose la construction d'un sentiment d'appartenance pour les habitants de diverses origines par la médiation de l'histoire locale, et prend les détours des valorisations du patrimoine dans le cadre de la politique touristique. La deuxième occurrence des habitants mentionne non pas leur réception mais leur intégration à ce qui est valorisé et signifié : l'enjeu est de « mieux comprendre la façon dont les gens vivent ", localement à Saint-Denis. Ils deviennent signifiés par la politique touristique.

Ce discours brouille les distinctions communes, entre touristes et habitants. À qui s'adressent alors les politiques de valorisation patrimoniale et touristique en périphérie? Elles ont en commun avec des politiques menées ailleurs le mot « tourisme » mais n'ont pas le même destinataire.

Le nouveau Schéma touristique communautaire de Plaine Commune 2012-2016 précise dès sa préface, rédigée par un élu communautaire (Ch. Mezerette), les destinataires de cette politique : "Plaine Commune accueille une population en difficulté, paupérisée davantage en période de crise, une jeunesse victime du chômage. Si le tourisme ne peut pas apporter des réponses à l'ensemble de ces problèmes, il peut toutefois contribuer de manière significative au développement de l'activité économique et de l'emploi. ». Le premier motif mentionné pour justifier la politique touristique concerne les retombées pour sa population. Il s'agit d'une justification par les résultats escomptés, qui construit la figure des bénéficiaires de façon large. C'est le schéma classique de l'action publique dans des territoires en difficulté.

Les dernières lignes de cette préface mentionnent également les publics de cette mise en tourisme: "L'enjeu est de taille: développer un tourisme pour tous, touristes étrangers, français, franciliens, et avec tous, salariés, étudiants et habitants en tant qu'acteurs et ambassadeurs; s'inscrire dans une volonté de développement local durable, générateur de retombées économiques, sociales et culturelles tangibles». Les habitants y sont donc mentionnés de différentes façons. En tant que population, ils sont les bénéficiaires ultimes. En tant qu'habitants ils sont partenaires, au double niveau d'«acteur » et d'«ambassadeur». Enfin, en tant que «franciliens», ils sont inclus dans les destinataires directs de la politique touristique, pris dans ce «tous", qui brouille les frontières entre catégories. "Les habitants" sont la réponse partielle ou totale aux questions : "pour qui ? ", « avec qui ? », « au bénéfice de qui ? » mener une politique de valorisation touristique du patrimoine.

40 Ainsi les mêmes habitants sont désignés par une multiplicité de catégories, pour mieux caractériser les modalités de la mobilisation et participation habitante dans le cadre de la mise en patrimoine en banlieue. Le prisme touristique est-il réducteur? Au contraire, l'hypothèse ici est celle d'une solidarité intrinsèque entre patrimonialisation et valorisation vers des publics, dont les touristes constituent l'archétype (mais pas la 
majorité). La figure de l'habitant s'y rencontre de multiples façons. Les politiques menées à Plaine Commune dans les années 2000 et 2010 figurent les habitants conjointement comme témoins, touristes, ambassadeurs, et co-producteurs.

\section{Les habitants comme témoins}

41 La construction patrimoniale mobilise tout d'abord les habitants comme témoins, dans le passage d'un patrimoine surtout monumental (la Basilique) à un patrimoine élargi, renvoyant à l'histoire industrielle et sociale locale. L'opération emblématique de cette figuration est l'exposition Douce banlieue, montée par le Service des Archives de SaintDenis en 2004, conçue comme collecte d'une mémoire ouvrière et populaire, à partir de témoignages oraux.

De façon similaire, différents dispositifs mémoriels sont chargés de collecter ces histoires des gens, et alimentent des projets patrimoniaux. La valorisation de la citéjardin de Stains s'appuie sur des entretiens menés avec les habitants, sur leur histoire résidentielle, devenant matériaux sonores d'une valorisation patrimoniale. L'association Mémoire Vivante de La Plaine, formée en 1996 pour constituer une mémoire d'un territoire industriel en transformation, procède également par collecte de témoignages. Son président, Jacques Grossard, est l'ancien directeur de Plaine Renaissance puis Plaine Commune, auteur également d'un guide sur les communes de Plaine Commune, repris par les acteurs institutionnels. De même, l'Unité patrimoine et arts visuels de la Courneuve mène des campagnes de collecte autour de l'histoire maraîchère et agricole qui concerne les communes de la Communauté d'agglomération, ainsi que les ouvriers des usines du territoire. Enfin, une collecte des mémoires immigrées est envisagée, tandis que les initiatives touristiques associatives encouragées par les acteurs locaux sur le thème des migrations, telle la balade de « la route des Indes à la Courneuve », mobilisent des témoignages locaux, sollicités auprès des commerçants par exemple (Chapuis, Jacquot, 2015).

Ces collectes sont une reconnaissance institutionnelle de l'importance des histoires vécues, d'une autre façon, par rapport aux décennies précédentes sur ce territoire, de faire patrimoine et de le valoriser. Elle est transversale aux diverses institutions en charge du tourisme et du patrimoine.

\section{Les habitants comme touristes}

Les habitants ne sont pas seulement témoins et figurants des histoires patrimoniales. Ils sont également appelés à vivre différemment ces espaces. La figure du touriste est mobilisée pour dire une nouvelle façon d'habiter et de découvrir son territoire. Une injonction douce à être touriste sur son propre territoire est énoncée.

Cela repose la question des destinataires des politiques de valorisation patrimoniale et touristique. La politique publique est alors plus complexe que dans les années 1970-1980, à l'époque du syndicat d'initiatives, qui séparait les objectifs de l'émission touristique (envoyer des Dionysiens vers d'autres destinations) de la réception touristique (attirer des touristes à Saint-Denis). Cette claire distinction fonde la séparation entre touristes et habitants. Or les politiques actuelles entrainent un brouillage dès lors que les habitants sont appelés à devenir touristes sur leur propre territoire. 

qui à la fois définit une échelle de référence de la signification patrimoniale (la banlieue, Plaine Commune, la ceinture maraichère, etc.), et vise à produire une reconnaissance par les habitants eux-mêmes de la justesse de cette référence pour dire leur rapport au territoire, par le biais des dispositifs touristiques de la valorisation patrimoniale. Cette figure de l'habitant-touriste est un détour, un moment nécessaire, pour favoriser cette réappropriation par l'habitant.

\section{Les habitants comme ambassadeurs}

51 En 1979, la directrice du syndicat d'initiatives considérait les touristes (au sens classique) comme les meilleurs ambassadeurs du territoire. Ce terme connaît à présent un retournement radical, puisque ce terme est désormais utilisé pour qualifier les habitants, encouragés par des dispositifs type Greeters (c'est-à-dire des habitants développant des visites de leurs lieux du quotidien, gratuitement) à faire la promotion de leur lieu de vie. 
52 Ainsi pour la coupe du monde de rugby de 2007, un guide du volontaire avait été mis en place. De même de nombreux documents évoquent les habitants comme ambassadeurs. Récemment, un accord a été monté avec Parisiens d'un jour pour structurer l'offre de Greeters, en lien avec le Comité Départemental du Tourisme de Seine-Saint-Denis et Plaine Commune. Ces Greeters sont donc en lien étroit avec les institutions publiques, qui contribuent à structurer ce mouvement, et proposent des formations.

53 Le développement de ces ambassadeurs du territoire constitue une synthèse des figures précédentes de l'habitant comme témoin, et de l'habitant se réappropriant son territoire par le détour du tourisme.

\section{Les habitants comme co-producteurs}

Cette structuration des Greeters et l'accent mis sur les habitants comme ambassadeurs font émerger une nouvelle figure, celle des habitants qui ne seraient pas seulement destinataires et engagés dans les politiques de valorisation, mais aussi co-producteurs d'une valorisation patrimoniale.

Franciade emblématise cette intégration des habitants à un projet patrimonial découlant de politiques institutionnelles. Cette association a été créée en 2002 pour développer des dispositifs articulant archéologie et appropriation locale, prolongeant les actions menées par l'Unité d'archéologie de Saint-Denis. Franciade vise le développement d'une filière locale à partir des fouilles, pour fabriquer des produits dérivés locaux, et permettre une meilleure connaissance du territoire local. La production des objets et souvenirs qui disent le territoire (reproductions libres d'objets issus des fouilles archéologiques, produits dérivés de la cité-jardin, etc.), est faite par des habitants disposant de savoir-faire, éventuellement issus des migrations qui ont constitué la ville : D. Melvan, tourneuse en céramique de Split, A. Rhadbane, brodeuse au Maroc. "L'association valorise les savoir-faire des habitants", "en faisant produire ces objets par des habitants en situation d'exclusion, grâce à la recherche des savoir-faire qu'ils détiennent » (Christnacht, 2011). Cette expérience pourrait être interprétée comme la reconnaissance d'un patrimoine immatériel, composant le patrimoine de banlieue. Mais il s'agit surtout de l'institution d'un rapport au territoire pour ses habitants, à travers la reconnaissance des compétences de ses habitants, mises au service d'une valorisation du territoire.

Les habitants sont ainsi figurés de diverses façons par les politiques de valorisation patrimoniale, dans différents registres d'action, en tant que destinataires, témoins, touristes, ambassadeurs et co-producteurs de ces mêmes politiques. Ces figures représentent les façons dont les habitants sont interpellés et catégorisés par les politiques de valorisation patrimoniale, par le détour d'un brouillage dans les discours de la distinction touristes - habitants. Des études et données manquent pour identifier l'efficacité de ces figurations, les participations effectives à l'action publique patrimoniale, ou le profil des habitants s'y inscrivant. Est-ce corrélé à la protogentrification de ces espaces, comme le montre L. Raad pour les fêtes locales (2012) attirant la classe moyenne et des nouvelles couches sociales, à l'instar des premiers temps du syndicat d'initiatives ? Pour la cité-jardin, sur les 3500 habitants, plusieurs centaines d'habitants de la commune ont pris part à une activité (visite, etc.), mais seuls quelques-uns participent de façon régulière à l'ensemble des activités d'animation 
patrimoniale. Le questionnement peut alors être retourné : comment cette intégration des logiques habitantes modifie-t-elle aussi l'action de valorisation patrimoniale?

\section{Porosités de l'action patrimoniale}

57 L'ensemble des figures habitantes évoquées maintiennent intactes la distinction institutions/habitants. Or les politiques de valorisation patrimoniale révèlent diverses porosités, qui concernent la logique de l'action patrimoniale, mais aussi les séparations habitants/institutions, à travers un rapprochement de leurs modalités d'action.

\section{Porosités entre logiques patrimoniales et urbaines}

58 Les politiques de valorisation patrimoniale ne relèvent pas du seul registre patrimonial. Le détour par les figures habitantes montre les enjeux symboliques sous-jacents, en termes d'image du territoire et de promotion d'un sentiment d'appartenance. La logique patrimoniale croise les enjeux d'aménagement et de requalification urbains, comme l'illustre le cas de la cité-jardin de Stains. En effet, ce projet emblématique de Plaine Commune, mentionné dès son premier Schéma Touristique Communautaire, constitue en même temps une opération de rénovation urbaine d'un quartier de logement social, menée dans le cadre de la politique de l'ANRU, avec comme acteurs principaux l'OPH 93 (bailleur social et propriétaire de la cité-jardin), Plaine Commune et la commune de Stains.

Plusieurs actions traduisent ces mélanges entre registre patrimonial et registre de la rénovation. La chargée de mission "valorisation touristique» pour la cité-jardin participe à des réunions dans le cadre de la rénovation urbaine, avec d'autres acteurs et des habitants. Cela permet une intégration de la thématique patrimoniale au projet de façon plus large, et en retour une imprégnation par les autres acteurs de l'intérêt d'une démarche de valorisation auprès des habitants (par exemple au motif que la réappropriation entraîne une diminution des incivilités).

60 L'intervention urbaine elle-même compose avec les motifs patrimoniaux : la cité-jardin est inscrite en 1976 à l'inventaire des sites, et dès lors soumise à l'avis de l'Architecte des Bâtiments de France pour toute transformation. Les cœurs d'îlots, partiellement à l'abandon, ont fait l'objet d'une réflexion dans le cadre de cette opération de rénovation urbaine, pour définir leurs usages, qui peuvent sembler contradictoires, entre attentes en termes de qualité de vie (usage comme espace sportif, prôné par le " comité des mamans ») et fidélité à l'esprit cité-jardin avec des usages maraîchers. Des dispositifs sont mis en place pour collecter les paroles habitantes, par exemple via le Collectif Cochenko, justifiant un aménagement différencié de chaque cœur d'îlot, entre usage maraîcher et usages publics. Les balades et animations menées dans la cité-jardin constituent aussi des façons d'encourager les habitants à se réapproprier certains de ces espaces. La porosité concerne là les objectifs des politiques de valorisation.

\section{Mise en cohérence des initiatives habitantes}

61 Des initiatives habitantes sont menées sur le territoire de façon plus déconnectée dans leur origine des politiques publiques. L'association Accueil Banlieues, née en 2010 dans le nord-est parisien, regroupe des habitants pratiquant l'hébergement à bas coût et des 
visites de découverte du territoire pour des visiteurs. L'objectif n'est pas économique mais militant, inspiré de la démarche Accueil Paysans, utilisant le tourisme pour faire découvrir un patrimoine ordinaire.

Toutefois l'articulation s'opère là a posteriori, via des " educ-tours ", qui s'adressent de façon large aux acteurs du tourisme : guides, archivistes, nouveaux salariés, mais aussi associations et habitants. Les visites sont thématisées, réalisées en lien avec la production d'une offre touristique à Plaine Commune. Il s'agit d'un dispositif qui produit de la cohérence dans les modalités de valorisation patrimoniale publiques et privées, par le partage d'un cadre commun de connaissances, entre acteurs d'horizons différents. Ces démarches permettent de construire l'idée d'une valorisation territoriale unifiée, de fédérer des acteurs via un portail (celui du CDT ou de l'OT). Accueil Banlieues participe à ces "éduc-tours" et tant les visites proposées que les thèmes mis en avant rejoignent les actions de valorisation patrimoniale menées par les institutions publiques.

\section{Engagements publics-habitants et Convention de Faro}

La mise en avant de l'habitant comme participant et co-producteur de l'action de valorisation patrimoniale transforme en retour les logiques d'action au sein des institutions, et illustre d'autres types de porosités.

En novembre 2013 est proposée à Saint-Denis une journée consacrée à la Convention de Faro, organisée par l'association d'habitants Accueil Banlieues, pour évoquer ses éventuels apports à la politique patrimoniale et touristique en banlieue, en présence de la coopérative sociale marseillaise Hôtel du Nord. La Convention de Faro, réalisée par le Conseil de l'Europe en 2005, évoque « la valeur du patrimoine culturel », le droit de chacun au patrimoine, et la notion de communauté patrimoniale, qui «se compose de personnes qui attachent de la valeur à des aspects spécifiques du patrimoine culturel qu'elles souhaitent, dans le cadre de l'action publique, maintenir et transmettre aux générations futures ». La Convention de Faro constitue ainsi un document énonçant à la fois la dimension libre et individuelle des choix patrimoniaux, déconnectés de toute assignation identitaire, et la façon dont peuvent être conduites des actions patrimoniales. Hôtel du Nord s'appuie sur la Convention de Faro pour justifier son engagement en faveur d'un patrimoine non nécessairement reconnu, notamment dans les quartiers nord de Marseille, et qui utilise les codes du tourisme (hébergement chez l'habitant, balades urbaines et visites guidées) pour sensibiliser sur la valeur sociale de ce patrimoine et permettre un développement solidaire de ces quartiers. L'organisation de cette journée en SeineSaint-Denis a donc pour enjeu la discussion et la diffusion des thèmes véhiculés par la Convention de Faro et Hôtel du Nord. En journée, des visites des quartiers urbains de la banlieue parisienne sont menées par des habitants (Greeters ou membres d'Accueil Banlieues), et la soirée est consacrée aux échanges, au théâtre de la Belle Étoile, à SaintDenis.

Cette soirée manifeste dans son déroulé même les porosités entre action publique et mobilisations citadines. Le décor est celui d'un théatre militant : à la Belle Étoile, un drapeau rouge flotte sur la scène, et en bruit de fond sonnent les chants latino de la fanfare qui répète dans une arrière-salle. L'espace est organisé selon un principe d'égalité, avec les chaises en rond, sans hiérarchie apparente. Une trentaine de personnes sont présentes, engagées à divers titres dans le patrimoine et le tourisme : 
des membres d'Accueil Banlieues (organisateurs) et d'Hôtel du Nord, des Greeters du territoire (Saint-Ouen, Saint-Denis), un membre du CA de Franciade, des membres des CDT 93 et $94^{13}$, des membres de différents services de Plaine Commune (économie sociale et solidaire, tourisme, etc.), des représentants d'associations, et quelques universitaires. Des membres des institutions publiques ayant un rôle important dans la valorisation patrimoniale et touristique sont présents, sans que cette appartenance soit laissée de côté : des brochures sur les projets touristiques et patrimoniaux de Plaine Commune sont présentes sur un étal à l'entrée, manifestant le sentiment de continuité entre ces initiatives habitantes et l'action publique. Toutefois une démarcation est construite dans les discussions, à travers des affirmations comme «on peut parler librement ", "on ne fait pas de politique ». Cette démarcation ne sépare pas les institutions des mobilisations habitantes, mais associe aux logiques volontaires et engagées des habitants celles des salariés des institutions, qui poursuivent par des voies institutionnelles des objectifs vécus comme similaires, et dont les conditions de possibilité sont définies par les élus, décideurs en dernier ressort. Les discussions portent sur la Convention de Faro, ses conséquences pour la valorisation patrimoniale et touristique, et la place des habitants dans ces actions. Des convergences apparaissent entre les participants, habitants et institutionnels, qui mettent en avant l'importance de l'entrée "tourisme " pour ce territoire de banlieue, aussi bien symboliquement qu'en termes de développement. Finalement le décalage apparaît davantage entre militants et institutionnels franciliens d'une part, et représentants d'Hôtel du Nord: pour ces derniers le tourisme est un prétexte pour attirer l'attention, plus qu'une activité à développer en soi.

\section{De nouvelles formes de l'action publique?}

66 La journée consacrée à la Convention de Faro illustre des formes de porosité entre institutions et initiatives habitantes, mais qui ne se limitent pas à de telles rencontres. Le fonctionnement même de l'action institutionnelle de valorisation patrimoniale intègre de façon principielle une porosité entre action publique et engagement citadin.

67 Il ne s'agit pas là d'évoquer des formes de récupération a posteriori des logiques militantes, telle par exemple l'intégration des friches à des politiques culturelles d'attractivité (Andres, Grésillon, 2013), mais d'envisager l'hypothèse d'une intégration a priori aux politiques patrimoniales publiques des logiques non institutionnelles qui caractérisent les démarches militantes, artistiques et habitantes. Le développement de la cité-jardin de Stains emblématise cela: présence sur le lieu directement, via un centre d'interprétation, tissage de relations personnalisées et quotidiennes avec les habitants, expérimentations plutôt que politiques généralisées, logique du happening et de l'événementiel, articulation avec des logiques artistes, parcours de la chargée de mission elle-même. Cette porosité de l'action publique à d'autres modalités d'intervention est permise aussi par le recrutement de nouveaux profils au sein des institutions, expliquant la présence d'acteurs publics multi-casquettes, également présents dans des trajectoires militantes, perçues en complément de l'action publique.

68 Alors qu'une étude des discours montre que les habitants sont interpellés par l'action publique à participer, un renversement est également perceptible avec l'internalisation à l'action publique des logiques d'action caractéristiques de l'engagement local. 


\section{Conclusion}

$\mathrm{Au}$ final, tranchant avec l'action touristique des années 1960 aux années 1980, les politiques publiques de valorisation patrimoniale touristiques menées à Plaine Commune reconfigurent les habitants de façon multiple, définissant leurs places et rôles. Bénéficiaires, témoins, ambassadeurs, participants, les mêmes habitants sont interpellés de multiples façons par l'action publique de valorisation patrimoniale, qui en les figurant désignent aussi un rapport au territoire et ses institutions, sur les plans discursifs (symboliques) et pratiques (engager des actions). Ces figurations accompagnent les territoires construits ou indiqués par ces politiques de valorisation patrimoniale, en lien avec les nouveaux enjeux patrimoniaux (patrimoine industriel, social, immatériel, agricole, etc.), et leur congruence ou désarticulation avec d'autres enjeux, notamment métropolitains (place de Plaine Commune au sein du Grand Paris, façonnage du Grand Paris culturel et touristique), renvoyant à une des orientations générales indiquée par le SCOT de Plaine Commune, approuvé en 2006 et révisé en 2009 : «Organiser le territoire sur son identité de banlieue populaire ». La récente élaboration du Contrat de Développement Territorial «Territoire de la création et de la culture », dans le cadre du Grand Paris, plaçant au cœur du développement les dynamiques numériques et créatives, pose la question de façon renouvelée, s'inquiétant d'une "situation contrastée entre le développement du territoire et la situation des habitants " ${ }^{14}$. Quelles seront les conditions du maintien de ces politiques de figuration et qualification du territoire, avec la constitution du Grand Paris prévue pour 2016, et le devenir incertain de Plaine Commune ${ }^{15}$ ?

Ces figurations révèlent aussi un étiolement des séparations habitant - institutions, par l'internalisation les logiques de l'engagement local à l'action publique elle-même, et donc une porosité croissante et un brouillage des modalités d'engagement entre action publique et mobilisation civique. Toutefois ce qui relève de des actions des habitants ordinaires eux-mêmes, sous la forme de l'adhésion, des modalités d'engagement, ou des éventuelles résistances, est resté en dehors de cette exploration. La question de la figuration des habitants, collectifs et publics par les politiques publiques patrimoniales peut être poursuivie pour identifier la façon dont ces collectifs figurés prennent corps, résistent, et transforment l'action publique et territoriale, voire imposent de nouvelles figurations.

\section{BIBLIOGRAPHY}

Andres L., Grésillon B., 2013. Cultural brownfields in European cities: a new mainstream object for cultural and urban policies. International Journal of Cultural Policy, 19-1, p. 40-62.

Anquetin V., Freyermuth A. (dir.), 2009. La figure de l'" habitant ». Sociologie politique de la "demande sociale". Presses Universitaires de Rennes, $184 \mathrm{p}$. 
Ashworth G., Tunbridge J., Graham B., 1999. A geography of heritage, power, culture and economy. London, Arnold.

Bacqué M.-H., Rey H., Sintomer Y. (dir.), 2005. Gestion de proximité et démocratie participative, une perspective comparative. La Découverte, $314 \mathrm{p}$.

Bellanger E., Mischi J. (dir.), 2013. Les territoires du communisme. Élus locaux, politiques publiques et sociabilités militantes. Armand Colin, Paris, 303 p.

Bertho A, 2008. La plaine Saint-Denis dans l'entre-deux. Projet, 2, p. 23-30.

Biland É., 2006. La « démocratie participative » en « banlieue rouge ». Les sociabilités politiques à l'épreuve d'un nouveau mode d'action publique. Politix, 2006/3, n 75 , p. 53-74.

Braouezec P., 2012. Mais où va la ville populaire?, entretien avec Jean Viard. Editions de l'Aube, 128 p. Brunet J.-P., 1980. Saint-Denis, la ville rouge : socialisme et communisme en banlieue ouvrière, 1890-1939. Hachette, $462 \mathrm{p}$.

Cefaï D., 2007. Pourquoi se mobilise-t-on? Les théories de l'action collective. La Découverte, 727 p.

Chapuis A., Jacquot S., 2015. Le touriste, le migrant et la fable cosmopolite. Mettre en tourisme les présences migratoires à Paris. Hommes et Migrations - Les Paris des migrants, Lazaridis M. et Weber S. (coord.), n 1308, décembre, p. 75-86.

Chavanon M., 2014. La mise en récit participative du passé migratoire local : le défi d'une mémoire partagée, entre projet urbain et mobilisation politique. Participations, 2014/3, $n^{\circ} 10$, p. 39-59.

Chivallon Ch, 2002. La mémoire maritime sélective des cités portuaires ayant pratiqué la traite des Noirs. In Péron F. (dir.), Le patrimoine maritime. Presses universitaires de Rennes, p. 341-344.

Christnacht M., 2011, Franciade recrée les objets du passé pour faire vivre le 93 au présent. Revue espaces, 292, p. 39-42.

Cousin S., 2007. L'argument du développement local par le tourisme. Actes de la Recherche en Sciences Sociales, $\mathrm{n}^{\circ} 170, \mathrm{p} .11-13$.

Decelle X., Jacquot, S., 2014. Coalitions publiques-privées et stratégies d'investissement hôtelier dans la constitution de la métropole touristique francilienne, entre centralités et périphéries. In Gravari-Barbas, Fagnoni, Métropolisation et tourisme. Comment le tourisme redessine Paris. Belin, Mappemonde.

Diçek M., 2007. Badlands of the Republic, Space, Politics and Urban Policy. Blackwell Publishing, 219 p.

Duchêne F., Langumier J., Morel Journel Ch., 2013. Cités ouvrières et patrimonialisation : d'un modèle à ses multiples transformations. Espaces et sociétés, 2013/1, n 152-153, p. 35-50.

Fabre D., 2013. Le patrimoine porté par l'émotion, In Fabre (dir.), Émotions patrimoniales. Editions de la Maison des Sciences de l'Homme, Ethnologie de la France, cahier 27, p. 13-98.

Fourcaut A., 2000. Pour en finir avec la banlieue. Géocarrefour, vol. 75, nº 75-2, p. 101-105.

Fourcaut A., 2007. « Les quartiers populaires ont aussi une histoire », Projet, 2007/4, 299, p. 7-15.

Fourniau J.-M., 2007. « Citoyen en tant que riverain » : une subjectivation politique dans le processus de mise en discussion publique des projets d'aménagement, In Blatrix et al., Le débat public, une expérience française de démocratie participative. La Découverte, Recherches, p. 67-77.

Glevarec H., Saez G., 2002. Le patrimoine saisi par les associations. La documentation française, 412 p. 
Gravari Barbas M., Jacquot S., 2014. Introduction, Patrimoine, tourisme, développement, une triangulation impossible. In M. Gravari-Barbas, S. Jacquot (dir.), Patrimoine mondial et développement au défi du tourisme durable. Presses de l'Université du Québec, p. 1-26.

Guillot X. (dir.), 2008. Firminy, Le Corbusier en héritage. Publications de l'Université de SaintEtienne, $211 \mathrm{p}$.

Jacquot S., 2009. Les figures de la ville, éléments de compréhension des débats sur la ville, à Gênes et Valparaiso. In C. Vallat, F. Dufaux, S. Lehman-Frisch (coord.), Pérennité urbaine, la ville par-delà ses métamorphoses. volume 3, Essence, Éditions de l'Harmattan, Paris.

Jacquot S., Gravari-Barbas M., Fagnoni E., 2014. Patrimonialisation et tourisme dans la région métropolitaine parisienne. Le patrimoine, clé de métropolité touristique? In M. Gravari-Barbas et E. Fagnoni, Métropolisation et tourisme. Comment le tourisme redessine Paris. Belin, collection Mappemonde, p. 103-117.

Kouloumbri Th., 2004. Plaine Commune, à la rencontre de la population locale. Revue espaces, 216, p. 29-32.

Lazzarotti O., 2004. Les nouvelles frontières du monde. Revue Espaces, tourisme \& loisirs, « Tourisme et banlieue ", $\mathrm{n}^{\circ} 216$, juin 2004, p. 14-17.

Le Goff J. (dir.), 1998. Patrimoine et passions identitaires, Actes des entretiens du patrimoine. Fayard, Éditions du patrimoine, $445 \mathrm{p}$.

Le Floch S., 2011. Le riverain, le citoyen et l'habitant : trois figures de la participation dans la turbulence éolienne. Natures Sciences Sociétés, 2011/4, vol. 19, p. 344-354.

Leniaud J.-M., 1996. Saint-Denis de 1760 à nos jours. Gallimard, archives, 361 p.

Malezieux J., 2003. Landy-France : un pôle d'activités dans la Plaine Saint-Denis. Nouvelles dynamiques de l'immobilier de bureaux en Ile de France au début des années 2000. Géocarrefour, vol. 78/4, p. 313-321.

Nicolas A., Zanetti T., 2013. Patrimoine et projet urbain : produire et valoriser la localité à SaintÉtienne, Nantes et Clermont-Ferrand. Espaces et sociétés, 2013/1, n 152-153, p. 181-195.

Noyer J., Raoul B., 2008. Concertation et « figures de l'habitant » dans le discours des projets de renouvellement urbain. Études de communication, 31.

Orellana L., 2006. Société civile, secteur privé : les nouveaux acteurs de la concertation dans les centres historiques de l'Amérique Latine et des Caraïbes. L'information géographique, 2006/2, vol. 70, p. 46-62.

Pecqueux A., Tornatore J.-L., 2013. Morale et politique dans le monument historique. L'incendie du château de Lunéville. In D. Fabre (dir.), Émotions patrimoniales. Éditions de la MSH, Cahiers d'ethnologie de la France, p. 283-310.

Petit G., 2014. Participations ordinaires et extraordinaires. Des appropriations différenciées d'une offre institutionnelle de participation municipale. Participations, 2014/3, n 10, p. 85-120.

Poulot D., 2006. Une histoire du patrimoine en Occident : XVIII-XXI siècle, du monument aux valeurs. Puf, le nœud georgien, $192 \mathrm{p}$.

Poupeau F.-M., 2009. Faire du patrimoine bâti un objet de concertation, l'expérience des Protections Ville de Paris. Revue française de sociologie, 2009/1, vol. 50, p. 123-150.

Raad L., 2012. Pratiques et représentations des couches moyennes de banlieue rouge : stratégies résidentielles et ancrage territorial. Espaces et sociétés, 148-149, p. 91-110. 
Rancière J., 2000. Le partage du sensible, Esthétique et politique. La Fabrique éditions, $74 \mathrm{p}$.

Rancière J., 2004. Aux bords du politique. Folio essais, 262 p.

Rancière J., 2008. Le spectateur émancipé. La Fabrique éditions, 145 p.

Rautenberg M., 2003. La rupture patrimoniale. Editions À la croisée, 173 p.

Robin L., 2013. Le label Villes et Pays d'Art et d'Histoire, outil de coopération des acteurs territoriaux au service du projet touristique à l'heure du Grand Paris ? Mémoire de Master 2 Tourisme, Université Paris 1 Panthéon Sorbonne.

Sherlock K., 2001. Revisiting the concept of hosts and guests. Tourist Studies, vol. 1, no 3, p. 271-295.

Sintomer Y., Blondiaux L., 2002. L’impératif délibératif. Politix, 15, 57, p. 17-35.

Stock M., 2005. Les sociétés à individus mobiles: vers un nouveau mode d'habiter?

EspacesTemps.net, http://www.espacestemps.net/document1353.html

Tomas F., 2004. Les temporalités du patrimoine et de l'aménagement urbain. Géocarrefour, 79/3, p. $197-212$.

Veschambre V., 2007. Patrimoine : un objet révélateur des évolutions de la géographie et de sa place dans les sciences sociales. Annales de géographie, 656, p. 361-381.

Veschambre V., 2008. Traces et mémoires urbaines. Presses Universitaires de Rennes, 315 p.

Violier, Ph., 2008. Tourisme et développement local. Paris, Belin, 192 p.

\section{NOTES}

1. Menée avec Géraldine Djament-Tran.

2. Il est d'ailleurs intéressant de comparer les modalités de négociation en 1975 et 2013 : en 1975 le syndicat d'initiatives est en première ligne, tandis qu'en 2013 les acteurs politiques ont davantage la main.

3. Source : Archives de Saint-Denis, série 268 W 1 à 5.

4. Source : Archives de Saint-Denis, série 14AC24, rapport au Bureau municipal 1979.

5. Archives de Saint-Denis, $268 \mathrm{~W}$, Rapport sur les perspectives d'activité, 1979.

6. Source : Bulletin municipal de Saint-Denis, avril 1980.

7. Plaine Commune rassemble en outre Aubervilliers, Épinay-sur-Seine, La Courneuve (depuis 2005), L'Île-Saint-Denis, Pierrefitte-sur-Seine, Stains, Saint-Denis, Villetaneuse.

8. Autour de la coopérative Hôtel du Nord, cf la thèse de doctorat en cours de Yannick Hascoet.

9. L'Atlas du Patrimoine de Seine-Saint-Denis réalisé par le Conseil Général de Seine-Saint-Denis constitue un inventaire et outil documentaire en ligne sur le patrimoine présent sur le département.

10. On retrouve cette logique dans d'autres territoires de petite couronne : Boulogne-Billancourt, Bagnolet, etc.

11. Conférence nationale permanente du tourisme urbain, 2-3 juillet 2012.

12. Les citations proviennent d'enquêtes personnelles, ou de paroles collectées dans le cadre de travaux d'étudiants dont j'assurais la coordination ou direction, auprès de différents acteurs en charge du tourisme et patrimoine sur ce territoire, entre 2011 et 2014, aux niveaux communal et intercommunal. Ce qui importe est la cohérence globale qui en ressort, et illustre une certaine façon de considérer l'enjeu patrimonial en lien avec les habitants, d'où le choix de l'anonymat. 
13. Les CDT sont les Comités Départementaux du Tourisme. Ceux des départements de SeineSaint-Denis et du Val-de-Marne se positionnent en partie sur un tourisme alternatif, valorisant les ressources patrimoniales spécifiques des territoires.

14. Accord cadre Territoire de la culture et de la création, 2012, p. 23

15. Les EPCI doivent être supprimés en 2016, remplacés par des EPT, Etablissements Publics Territoriaux.

\section{ABSTRACTS}

Plaine Commune, institutional territory from the Parisian suburbs, in Seine-Saint-Denis (french department), is characterized by heritage valorisation policies, particularly for tourism development. However the meaning of these policies has evolved. For a long time a classical type of heritage is valorized, as a testimony of national history. During the 1990s, a new conception of local heritage emerges and is promoted, that reflects the popular and industrial history of the territory. These heritage public policies have a symbolic dimension, producing discourses on the inhabitants, and defining their roles and places. These inhabitants are categorized in different ways by heritage public policies: as beneficiaries, witnesses, ambassadors, participants, and even tourists. These figurations also reveal a blurring of the distinction between inhabitant and institutions, redefining the logics of heritage public policies.

Plaine Commune, banlieue parisienne en Seine-Saint-Denis, est marqué par des politiques de valorisation du patrimoine, notamment à des fins touristiques. Toutefois le sens de ces politiques a évolué. Pendant longtemps est valorisé un patrimoine classique, reflet de l'histoire nationale. Les années 1990 font rupture, valorisant un patrimoine qui témoigne de l'histoire populaire et industrielle de ce territoire. Cette action publique patrimoniale comporte une dimension symbolique, produisant des discours sur les habitants, et leur définissant des places et rôles. Ces habitants sont interpellés de différentes façons par l'action publique de valorisation patrimoniale:bénéficiaires, témoins, ambassadeurs, participants, et même touristes. Ces figurations révèlent aussi un brouillage de la distinction habitant - institutions, au cœur des logiques de l'action publique patrimoniale.

\section{INDEX}

Mots-clés: Plaine Commune, valorisation patrimoniale, banlieues, figures habitantes, tourisme Keywords: Plaine Commune, heritage valorisation, parisian suburbs, figures of inhabitants, tourism

Subjects: Sur le Champ - Sur le Terrain

\section{AUTHOR}

\section{SÉBASTIEN JACQUOT}

Sébastien Jacquot, Sebastien.Jacquot@univ-paris1.fr, est Maître de conférences en géographie à l'Université Paris 1 Panthéon Sorbonne.Il a publié récemment : 
- Chapuis A., Jacquot S., 2014. Le touriste, le migrant et la fable cosmopolite. - Mettre en tourisme les présences migratoires à Paris. Hommes et migrations, $\mathrm{n}^{\circ}$ 1308, décembre 2014, Les Paris des migrants, p. 75-84.

- Notarangelo C., Jacquot S., 2014. Discorsi sull'illegalità nel cuore turistico di Genova. Gli ambulanti senegalesi al Porto antico. In Luisa Faldini, a cura di, Dal Mediterraneo al Baltico. Dinamiche sociali in area urbana. Atti del Convegno, Genova, 23 ottobre 2014, Edizioni Epoké, Novi Ligure, p. 107-120.

- Gravari-Barbas M., Jacquot S., 2014. Introduction : Patrimoine, tourisme, développement, une triangulation impossible? In Gravari-Barbas, Jacquot (dir.), Patrimoine mondial et développement au défi du tourisme durable, Presses de l'Université de Québec, p. 1-26 